\title{
When should a coroner's inquest be held? The Manchester guidelines for pathologists
}

\author{
I S D Roberts, L M Gorodkin, E W Benbow
}

There are two main reasons for deaths to be reported to the coroner: either that the cause of death is unknown or that death was "violent or unnatural". The distinction between "natural" and "unnatural" death is not legally defined, and there is a grey area in which the decision to report a death to the coroner, the decision to hold an inquest, and the final verdict vary greatly according to the personal attitudes of both doctor and coroner.

In most deaths reported to the coroner, a necropsy will be performed to determine the cause of death. The coroner then decides to certify the death as a result of natural causes, or to open an inquest, largely on the basis of the cause of death provided by the pathologist. Although the final decision in difficult cases is made by the coroner, it is common practice for the pathologist, when providing a cause of death, to be asked to indicate whether, in his or her opinion, death was natural or otherwise. In our experience, however, there is considerable variation in the way different pathologists approach borderline cases. In many instances their approach differs from that of the coroner, and it is relatively unusual for coroners to communicate their views to clinicians and pathologists. This variation causes confusion for clinical staff and is a potential source of distress to relatives of the deceased. Recognising a need for greater consistency in the reporting of borderline cases, the Manchester Coroner and senior pathologists in central Manchester held a meeting to discuss the interface between natural and unnatural death. Using scenarios based on difficult cases from our personal experience, we had discussions with the specific objective of reaching a consensus and producing written guidelines for pathologists in Manchester. It is clear from a recent survey of the attitudes of coroners in England and Wales ${ }^{1}$ that there is also considerable variation in the way coroners approach borderline cases. Therefore, many will disagree with some of our conclusions, but it is our opinion that achieving consistency is most important, because there is no clear "right" or "wrong" approach to many of the issues raised. Here, we present the Manchester guidelines, together with some explanation of how we reached our conclusions. The deaths discussed fall in to four categories: (1) deaths resulting from infections, (2) deaths after surgery, (3) deaths related to medical treatment, and (4) deaths after fractures.

In most cases, a consensus view that death is the result of natural causes implies that a coroner's inquest is unnecessary. There are, however, exceptions to this general rule; sometimes, the precise circumstances of death are only determined at inquest, and a coroner might then be able to give a verdict of natural causes. The pathologist should indicate to the coroner if it is thought that an inquest should be held in a presumed natural death, to confirm this conclusion.

\section{Deaths resulting from infections}

The essential question posed is: "what is a natural route or source of infection?".

(1) Infection as a result of intravenous drug abuse. Example cause of death:

I (a) bacterial endocarditis

I (b) intravenous injection

I (c) drug abuse.

In all cases where death resulted from introduction of the infective agent by intravenous injection, death should be regarded as unnatural and an inquest opened. The verdict in most cases will be misadventure, rather than accidental, because injection was deliberate but had not been intended to introduce infection into the blood.

(2) Hepatitis B or C liver disease. Example cause of death:

I (a) hepatic cirrhosis

I (b) hepatitis $C$ virus infection

I (c) blood transfusion.

In instances where there is a history of blood transfusion or intravenous drug abuse, and where this was the probable route of infection, the death should be regarded as unnatural. In cases where no known risk factor exists for infection with either hepatitis B or C, the cause of death should be regarded as natural. It is unnecessary to hold an inquest to search for possible risk factors when these are not evident. In the postmortem report the pathologist should make the comment: "I am not aware of any risk factors for the hepatitis $\mathrm{B} / \mathrm{C}$ virus infection in this individual".

(3) Human immunodeficiency virus (HIV) deaths. The verdict depends upon the route of infection. In cases in which there is evidence of blood transfusion, injection of blood products or intravenous drug abuse, the death should be regarded as unnatural and an inquest opened. In those cases with no known risk factors, or in which there is evidence of a sexually transmitted route (homosexual or heterosexual), death should be regarded as natural.

(4) Ingestion of infected food. Deaths after ingestion of infected food or water may be regarded as natural. This includes agents that cause an acute diarrhoeal illness and Creutzfeld Jacob disease (CJD) linked to consumption of infected beef products. Example cause of death:
Accepted for publication 6 December 1999 
I (a) colitis

I (b) Escherichia coli 0157 infection

I (c) ingestion of infected meat.

In many cases there will be no obvious link between the infection and a particular food item, and therefore I (c) would be omitted. Regarding these cases as natural causes of death would not affect any public health investigations. If, however, the source of the infection is known and there is the possibility of neglect then an inquest should be opened to investigate the circumstances of infection.

(5) Sepsis as a result of pressure sores. Pressure sores can be viewed as a manifestation of severe debility and immobilisation, in much the same way as bronchopneumonia. If the underlying condition is natural-for example, cerebral infarction, then death as a result of sepsis secondary to pressure sores may also be viewed as natural. However, pressure sores are avoidable with appropriate levels of care and a verdict of "natural causes aggravated by neglect" might in some instances be appropriate. Because this verdict is dependent upon the circumstances of the death, and quality of care, an inquest is necessary to collect the available evidence. Therefore, most of these deaths will necessitate the opening of an inquest. Of course, if pressure sores follow immobilisation secondary to an injury, then the verdict will depend upon the circumstances of the injury.

\section{Deaths after surgery}

Although surgery or anaesthesia contribute towards death in most postoperative deaths, in many instances death would have occurred at the same time, or earlier, with no surgical intervention. The crucial question to ask in each case is "has the surgery accelerated death?".

(1) Death after emergency surgery for a life threatening condition. Example cause of death:

I (a) pulmonary embolism

I (b) deep vein thrombosis due to immobilisation

I (c) perforated duodenal ulcer (treated surgically).

These deaths can be regarded as natural, as long as the pathologist feels able to make the statement that: "there is no evidence at necropsy of operative mismanagement".

(2) Deaths after elective surgery. In those instances when there is a link between surgery/anaesthesia and the immediate cause of death (for example, postoperative bronchopneumonia, pulmonary embolism, or myocardial infarction) and death has been accelerated by the operation, an inquest is necessary. There remains the question of how long after surgery should complications such as bronchopneumonia be attributed to the operation. Rather than putting an arbitrary time point, such as one week or one month, the timing should be determined by the period of immobilisation. In most instances, this approximates to the period of hospital admission. Regarding postoperative myocardial infarction, the increased risk of suffering a myocardial infarct, given a certain degree of coronary artery atheroma, falls with increasing time after surgery. The absolute risk at each day after surgery is unknown and differs with each patient, depending on the circumstances. Therefore, pathologists should use their own discretion in deciding whether a postoperative myocardial infarct is related to surgery or not.

\section{Deaths related to medical treatment}

(1) Deaths associated with immunosuppression. In many instances of death as a result of infection after chemotherapy, the immunosuppression is a result of the chemotherapy, rather than the underlying disease process. For example, neutropenia after bone marrow transplantation for leukaemia. Example cause of death:

I (a) fungal pneumonia

I (b) acute myeloid leukaemia (treated with chemotherapy).

Many untreated malignancies, such as acute leukaemia, are rapidly fatal; therefore, although chemotherapy has resulted in death, it has not accelerated death. Thus, post-chemotherapy deaths for malignancy are acceptable as natural, as long as the pathologist makes the following two comments in his/her report: (i) treatment did not accelerate death, and (ii) treatment was given appropriately. Deaths resulting from complications of radiotherapy or chemotherapy, other than immunosuppression, are often avoidable and necessitate an inquest; these complications include conditions such as haemorrhage and radiation enteritis.

The situation of immunosuppression for organ transplantation is, however, less clear cut because, particularly for renal transplantation, an alternative treatment is available and a patient may survive many years on dialysis without a transplant. Therefore, in all cases in which death results from infection as a result of immunosuppression after organ transplantation an inquest should be held.

Splenectomy causes a specific type of immunosuppression, predisposing to pneumococcal infections. Death secondary to pneumococcal lobar pneumonia in a patient who has previously had a splenectomy requires an inquest; the event necessitating splenectomy, commonly traumatic haemorrhage, might have contributed towards death and a misadventure verdict might be appropriate.

(2) Haemorrhage after anticoagulation or thrombolysis. Death as a result of haemorrhage in a patient who has received anticoagulant or thrombolytic treatment for thrombotic events is potentially unnatural. An inquest should be held to determine the extent to which anticoagulation has contributed towards haemorrhage.

(3) Peptic ulceration in patients taking nonsteroidal anti-inflammatory drugs (NSAIDs). Example cause of death:

I (a) peritonitis

I (b) perforated duodenal ulcer

II arthritis treated with non-steroidal antiinflammatory drugs.

Treatment with NSAIDs, including aspirin, is an important risk factor for peptic ulceration. 
Thus, these deaths are potentially unnatural and require an inquest.

(4) Thromboembolism in women taking the oral contraceptive pill. Example cause of death:

I (a) pulmonary embolism

I (b) deep vein thrombosis

II prothrombotic tendency associated with the oral contraceptive pill.

Thrombotic deaths, in which the oral contraceptive pill is the only risk factor identified, require an inquest to investigate the precise role of the oral contraceptive pill; the thrombotic risk depends upon the oestrogen content of the pill and evidence collected at inquest might reveal other factors predisposing to thrombosis.

\section{Deaths after fractures}

In those instances where death follows traumatic fracture of healthy bones, an inquest is necessary and an accidental death verdict is usually appropriate. In the case of death after pathological fracture, the trauma might have been insufficient to produce a fracture in a person with previously healthy bones. As a result, it is the practice of many doctors and coroners to assign these deaths as natural. Example cause of death:

I (a) bronchopneumonia

I (b) fractured neck of femur

I (c) osteoporosis and fall.

However, even if there is evidence of pronounced pre-existing bone pathology, in most cases death is in part a result of a fall and, therefore, not entirely natural. An inquest should be held in all such cases to determine the circumstances of the fall. This applies also to fracture of bones weakened by malignancy. The exception to this is when there is clear evidence that the fracture preceded the fall, rather than resulted from it. An example, from our experience, is an old woman with osteoporosis who got out of bed on the ward, rotated on one leg to turn round, a loud crack was heard by nursing staff, and she fell over with a fractured neck of femur. As with postoperative deaths, death from bronchopneumonia or pulmonary embolism during the period of immobilisation should be regarded as resulting from the fracture and fall. Death occurring in an individual who has been fully mobilised and has gone home might be regarded as unrelated to the fracture and potentially a result of natural causes.

\section{General comments}

Potential side effects of drug treatment should be investigated at inquest. The true incidence of deaths related to therapeutic drugs will only be determined if all such deaths are investigated and documented accurately. In general, however, deaths related to medical or surgical treatment should only be regarded as unnatural if intervention has accelerated death, or if there is evidence of inappropriate treatment or surgical mishap. The attitude of the family or friends of the deceased might also have an impact. Although the family cannot force a coroner to open an inquest, many coroners indicate that if the family does express dissatisfaction with the care received, they might open an inquest, even if death is believed to be the result of natural causes. ${ }^{1-3}$

These guidelines are open to continual review and will evolve with changing attitudes in society. A major factor in determining whether a cause of death is viewed as natural or otherwise is how frequently a particular disease process causes death. With changing incidences of fatal diseases, the guidelines will inevitably change over time. For example, chronic alcoholic liver disease and bronchial carcinoma related to cigarette smoking are currently accepted as natural causes of death. The distinction of alcoholic cirrhosis from hepatic cirrhosis of viral aetiology, and of smoking related carcinoma from malignancy induced by environmental pollution or industrial carcinogens, is arbitrary and not entirely logical. Alcohol and cigarette smoking certainly pose a far greater hazard to the health of Western societies, but the very fact that they are so common prevents these deaths from being investigated through a coroner's inquest. Similarly, death as a result of ingestion of infected food and water is now considered unnatural by most coroners in England and Wales, ${ }^{1}$ despite this being one of the most common causes of death worldwide. This undoubtedly reflects the relative rarity of this cause of death in our society today, and the high expectations of a clean food and water supply. The recommendations made in the section on the ingestion of infected food attempt to take into account both public health concerns and also the fact that ingestion is a common and natural route of infection.

Guidelines on when to report a death to the coroner are included in the book of medical certificates of the cause of death (form 66), ${ }^{4}$ and have also been issued by the Office of National Statistics. Despite this, clinical staff appear to have a generally poor understanding of the coronial system, and in particular when to report deaths. ${ }^{56}$ This, in part, reflects deficiencies in medical education, but it might not be that surprising in view of the great variation in the attitudes of different coroners to what constitutes natural and unnatural death. ${ }^{1}$ Most pathologists assume that they have an excellent understanding of when a coroner's inquest is needed but, in our experience, they show an equally diverse approach to borderline cases. These difficult cases are common in hospital practice, especially when medical or surgical intervention may, in part, contribute towards death. There are no national guidelines to help pathologists deal with the interface between natural and unnatural death. Although we recognise that the Manchester guidelines will not achieve universal acceptance, we hope that they will form the basis for future discussions, and a national consensus.

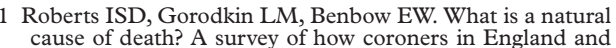
cause of death? A survey of how coroners in England and
Wales approach borderline cases. f Clin Pathol 2000;53:367-73. 
2 R v Poplar Coroner ex parte Thomas, The All England Law Report 1993(2):381-9.

Levine M, Pyke J. Levine on coroners' courts. London: Sweet and Maxwell, 1999.

4 Form 66. Forms for medical certificates of the cause of death under the Births and Deaths Registration Act 1953. London: HMSO, 1985
5 Start RD, Delargy-Aziz Y, Dorries CP, et al. Clinicians and the coronial system: ability of clinicians to recognise reportable deaths. BMF 1993;306:1038-41.

6 Start RD, Usherwood TP, Carter N, et al. General practitioners' knowledge of when to refer deaths to a coroner. Br F Gen Pract 1995;45:191-3.

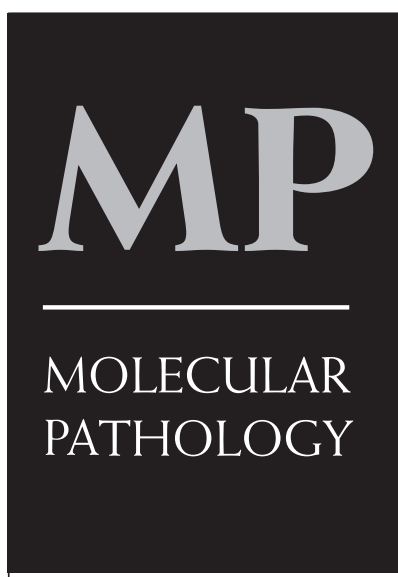

\section{Contents}

April 2000 Vol 53 No 2

\section{Reviews}

55 Apoptosis $S$ Afford, $S$ Randhawa

64 Laser capture microscopy SCurran, f A McKay, H L McLeod, G I Murray

\section{Papers}

69 Cellular localisation of HHV-8 in Castleman's disease: is there a link with lymph node vascularity? 7 O'Leary, $M$ Kennedy, D Howells, I Silva, V Uhlmann, K Luttich, S Biddolph, S Lucas, $\mathcal{F}$ Russell, N Bermingham, M O'Donovan, M Ring, $C$ Kenny, $M$ Sweeney, O Sheils, C Martin, S Picton, K Gatter

77 Characterisation of a mouse monoclonal anti-idiotype reactive with a $\mathrm{V}$ region sequence commonly used by human immunoglobulins $\mathcal{F} E \mathrm{McElveen}$, P B Furtado, S F Smith, M R Clarke, I Spendlove, H F Servell, F Shakib

83 Acute lymphoblastic leukaemia: correlation between morphological/ immunohistochemical and molecular biological findings in bone marrow biopsy specimens $S M$ Kröber, A Greschniok, E Kaiserling, H-P Horny

88 Genetic polymorphisms of cytochrome p4502E1 and susceptibility to alcoholic liver disease and heptocellular carcinoma in a white population: a study and literature review, including meta-analysis NACS Wong, F Rae, Kf Simpson, $G D$ Murray, $D \mathcal{F}$ Harrison

94 Identification of different Borrelia burgdorferi genomic groups from Scottish ticks $C$ L Ling, $A W$ L foss, $M M$ Davidson, $D$ O Ho-Yen

99 Proteolysis in human breast cancer EA Garbett, $M W R$ Reed, $T$ f Stephenson, $N$ F Brown

\section{Short report}

107 Detection of SYT-SSX fusion transcripts in both epithelial and spindle cell areas of biphasic synovial sarcoma using laser capture microdissection T Kasai, S Shimajiri, H Hashimoto 